\title{
Abstract concepts and the suppression of arbitrary episodic context
}

\author{
Charles P. Davis (charles.davis@uconn.edu) \\ Psychological Sciences, CT Institute for the Brain and Cognitive Sciences, \& Brain Imaging Research Center, \\ University of Connecticut \\ Pedro M. Paz-Alonso (p.pazalonso@ bcbl.eu) \\ Basque Center on Cognition, Brain and Language \\ Gerry T. M. Altmann (gerry.altmann@uconn.edu) \\ Eiling Yee (eiling.yee@uconn.edu) \\ Psychological Sciences \& CT Institute for the Brain and Cognitive Sciences, University of Connecticut
}

\begin{abstract}
Context is important for abstract concept processing, but a mechanism by which it is encoded and re-instantiated with concepts is unclear. We used a source-memory paradigm to determine whether episodic context is attended more when processing abstract concepts. Experiment 1 presented abstract and concrete words in colored boxes at encoding. At test, memory for the frame color was worse for abstract concepts, counter to our predictions. Experiment 2 showed the same pattern when colored boxes were replaced with male and female voices. Experiment 3 presented words from encoding in the same or different box color to determine whether a greater advantage is conferred by context retention in memory for abstract concepts. There was instead a disadvantage: abstract concepts were less likely to be identified when the encoding color was retained at test. Concrete concepts are more sensitive to simple episodic detail, and in abstract concepts, arbitrary context may be suppressed.
\end{abstract}

Keywords: concepts, semantic memory, episodic memory, abstract concepts, concreteness

\section{Introduction}

Abstract concepts like decision are central to the human experience, yet little is understood about how they are processed. Contextual information is thought to be important to abstract concepts-the specific meaning of decision varies more depending on context than does the meaning of river. While a river in New England shares many properties with a river in Papua New Guinea, consider the case of decision: your decision on which beverage to buy at a café late at night differs greatly from the decision a judge might make in determining sentencing for a felon. It is the context which determines the antecedents, outcomes, and consequences in these two instantiations of decision. While it seems that context should be important in processing abstract concepts, the mechanism by which context is encoded and reinstantiated with the concept remains unclear. One possibility is that the episodic memory system, which supports encoding and recall of contextually detailed memories, is critical in understanding abstract concepts. Thus, here we probed a potential mechanism underpinning abstract concepts' sensitivity to context by using a source memory paradigm to test whether episodic context is better bound to abstract than concrete concepts.

Episodic memory is classically defined as explicit memory for unique events (Tulving, 1983, 2002), where episodic context is the detail that colors an episode. There are circumstances under which we are more likely to encode, and therefore, recall the arbitrary contents of a particular episode (e.g., the color of a frame or the identity of a speaker). A standard paradigm for assessing this ability is the source memory task (see Davachi, 2006; Yonelinas, 2001, 2002). In this task, participants are asked at test to determine whether an item (e.g., a word) was previously presented in an exposure phase, and then probed as to whether they can recognize some prior contextual detail. Greater confidence in having seen a word at exposure is associated with greater likelihood of having encoded the contextual detail (e.g., Kirwan, Wixted, \& Squire, 2008; Yu, Johnson, \& Rugg, 2012). Therefore, we would predict that greater confidence in having seen or heard a word during an encoding phase is associated with better memory for an arbitrary context, such as a box color or voice, at a test phase.

In addition to confidence in recollection or strength of the memory, emotionality in words, including both valence and arousal (Kensinger \& Corkin, 2003), influences the likelihood of recalling the context in which something was presented, suggesting that the content of the stimuli at exposure can influence the likelihood that the context is identified at test. More specifically, conceptual or semantic content might affect likelihood of context encoding. In this set of experiments, we investigated whether this is true for concreteness: are we better at encoding contextual detail for abstract than for concrete concepts?

The notion that episodic context is more important for interpreting abstract concepts suggests that we should be more sensitive to the episodic context in 
which abstract concepts are placed and, in turn, be more accurate at retrieving even non-systematically related elements in the context. We opted to test this hypothesis by examining whether arbitrary contexts are better recognized when paired with abstract as compared to concrete concepts. Because memory is generally better for concrete than for abstract words (e.g., Paivio, Walsh, \& Bons, 1994), we expected that although overall memory for concrete concepts would be better, when abstract concepts are correctly recognized, the context would be better encoded. To foreshadow the results, we find evidence against this hypothesis, suggesting that arbitrary episodic context may be inhibited in abstract concepts. In the General Discussion, we propose an alternative framework in which these results might be accommodated.

In the studies below, context is operationally defined as an aspect of a stimulus that is irrelevant to the central stimulus, such as whether a target word is presented within a red or green frame or whether stimuli are presented in a male or female voice.

\section{Experiment 1}

\section{Methods}

Participants Forty-two University of Connecticut (UConn) students with normal or corrected-to-normal vision and hearing provided informed consent and received course credit for participating. One participant was excluded for non-compliance, leaving $N=41$. The study was approved by the UConn IRB.

Stimuli In the encoding phase, 100 (60 target, 40 nontarget) abstract (e.g., decision) and 100 (60 target, 40 non-target) concrete (e.g., chair) noun concepts were used. (Non-targets were synonym words which functioned as positive responses for the synonymjudgment task described below. Targets were nonsynonyms.) Stimuli were matched across all stimulus subsets on word length and word frequency based on English Lexicon Project data (Balota et al., 2007), and were sorted into abstract and concrete conditions based on Brysbaert, Warriner, and Kuperman's (2014) concreteness norms (Table 1). Half of the words were enclosed in red boxes, and the other half in green, and this was balanced across concrete and abstract words. In the test phase, an additional 50 abstract and 50 concrete words were added to the target and non-target items.

Table 1

Stimulus Characteristics

\begin{tabular}{lllllll}
\hline & \multicolumn{3}{c}{ Targets } & \multicolumn{3}{c}{ Synonyms } \\
\cline { 2 - 7 } & $\mathrm{n}_{\text {letter }}$ & $\log ^{\mathrm{F}}$ & conc & $\mathrm{n}_{\text {letter }}$ & $\log ^{\mathrm{F}}$ & conc \\
\hline Abs & 6.7 & 5.0 & 1.8 & 7.3 & 5.7 & 2.1 \\
Conc & 7.0 & 5.1 & 4.9 & 6.2 & 5.7 & 4.8 \\
\hline
\end{tabular}

Procedure Participants performed a two-phase source memory task. Stimuli were presented visually one at a time, in pseudorandomized order, with an arbitrary box context (either a red or a green box). On each word, participants performed a synonym-judgment 1back task. To ensure that they did not ignore the boxes, the hand they used to make their response was determined by box color (left hand for words in green boxes and right for red). Stimuli were presented for $2000 \mathrm{~ms}$ with a 1000-ms interstimulus interval. Participants were told there would be a later memory test on the words, but not that source (i.e., box color) memory would be tested.

In the test phase, participants performed two tasks for each word. First, they responded whether they had seen the word at encoding, indicating their degree of confidence in the decision (high, medium, and low confidence for either "old" or "new"). Second, for old words, they indicated the color of the box on initial encoding. The task was the same for new words, except that they were asked simply to select the color they thought the box would have been had it been presented at encoding. Participants were given 6000 ms each for the old/new and the box color judgment.

Data analysis Data were analyzed using R. Memory for items (i.e., words) and their contexts (i.e., box color) was first analyzed using descriptive statistics, calculating accuracy, hit rate, miss rate, correct rejections, false alarms, and d' (calculated as $z$ (Hit) $z$ (FA)) for all words, and accuracy was also assessed by level of confidence. Source (i.e., box) memory accuracy was calculated only for target hits, and was assessed across confidence levels. Source memory accuracy was analyzed as a function of word type and confidence in having seen the word at encoding. Logistic mixed effects models (lme4 package; Bates et al., 2017) were used to analyze the data, with subject and word as random intercepts, and word type (abstract or concrete), level of confidence (low, medium, high), and their interaction as treatmentcoded fixed effects. Each predictor was entered in a successive model, and statistical significance was assessed by comparing the models using likelihood ratio tests. Here, $p$-values $<.05$ were considered statistically significant.

\section{Results}

Item recognition First, to provide a baseline measure of memory for concrete and abstract words, we report the accuracy and hit, miss, correct rejection, and false alarm rates across all words (Table 2). Hit rates were higher and false alarms lower in concrete words, demonstrating the mirror effect (Glanzer \& Adams, 1985), which has previously been observed for concreteness (Glanzer \& Adams, 1990). For overall accuracy, there were main effects of both word type 
and confidence. Concrete words were better recognized than abstract $\left(\chi^{2}(1)=10.21, p=.001\right)$, and accuracy increased with greater confidence $\left(\chi^{2}(2)=\right.$ $571.37, p<.001)$. The interaction was non-significant. Among targets only (i.e., non-synonym words presented at encoding), there was no main effect of word type $\left(\chi^{2}(1)=0.29, p=.59\right)$, but a main effect of confidence level $\left(\chi^{2}(2)=675.22, p<.001\right)$, with words recognized better with higher confidence. The interaction was non-significant. Means and 95\% CIs for word and source (i.e., box) memory are shown in Figure 1. Finally, d' analysis showed that when considering response sensitivity, accuracy was better for concrete concepts, $t(39)=-5.37, p<.001$.

Table 2

Mean Item Recognition Accuracy

\begin{tabular}{lcccccc}
\hline $\begin{array}{l}\text { Word } \\
\text { type }\end{array}$ & Acc & Hit & Miss & CR & FA & d' \\
\hline $\begin{array}{l}\text { Abstract } \\
\text { Concrete }\end{array}$ & .73 & .77 & .23 & .65 & .35 & 1.21 \\
\hline
\end{tabular}

Note. $\mathrm{CR}=$ correct rejection; $\mathrm{FA}=$ false alarm.

Source memory Here, we included only trials for which the word had been correctly identified. There was a main effect of word, where the box was less likely to be remembered for abstract words $\left(\chi^{2}(1)=\right.$ $5.45, p=.02)$, but not of confidence level. The interaction was non-significant. Participants were less likely to correctly remember the box color for abstract words (Figure 1).

According to d' scores, there was a baseline advantage for recognizing concrete words, which would then bias the source memory models. Correct memory trials for abstract words may have been less likely to reflect true hits where the word was in fact encoded. Accordingly, we also constructed models with d' as a predictor. A likelihood ratio test comparing the model with both d' and word type versus the model with only $\mathrm{d}^{\prime}$ was significant, $\chi^{2}(1)=5.27, p=.02$, suggesting that the effect of word type, where box recognition was worse in abstract than it was in concrete concepts, was significant even after accounting for the d' concreteness advantage.

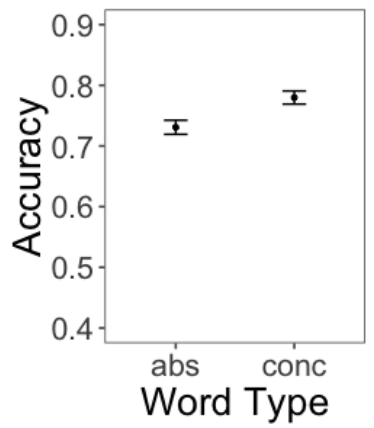

Figure 1. Effects of concreteness on (a) overall item recognition accuracy and (b) source (i.e., box) memory.

\section{Discussion}

Source memory was worse for abstract concepts, and this was true even after controlling for a concreteness advantage detected in $\mathrm{d}$. Thus, the results of Experiment 1 ran counter to our hypothesis: source memory was worse for abstract than for concrete words, even when participants were highly confident in having seen the word at encoding. Why did this unexpected difference emerge? It may be that concrete concepts are more amenable to a mnemonic strategy wherein a color adjective (i.e., "red" or "green") could readily be bounded to concrete objects (e.g., "table"), making source memory better for concrete words. Thus, it may be that contextual detail is better encoded in abstract concepts, but only when not systematically related to concrete objects (as may be the case for colored boxes). A second explanation is that counter to our main hypothesis, the concreteness advantage extends to memory for arbitrary contextual details. Experiment 2 evaluated these competing explanations.

\section{Experiment 2}

In Experiment 2, we utilized a variant of the source memory paradigm, where instead of the box, the context to be encoded was a male or female voice. Concepts were presented auditorily, and memory was assessed on visually presented words (e.g., Wilding \& Rugg, 1996). In line with the original prediction that contextual detail is encoded to a greater extent in abstract concepts, it was predicted that source memory (i.e., male or female voice) would be better for abstract concepts. This prediction is further buoyed by the finding that person-related social properties may be more important for abstract concepts (Barsalou \& Wiemer-Hastings, 2005).

\section{Methods}

Participants Forty-two UConn undergraduates with normal or corrected-to-normal vision who had not participated in Experiment 1 provided informed 
consent and were given course credit for their participation.

Stimuli The words were the same as those used in Experiment 1, but rather than being presented visually they were instead recorded by a male and a female speaker, with half the words presented by the male speaker and half by the female speaker. As with box color, this list was held constant across participants. There were no differences in the length of the sound files between the two speakers, and all files were normalized to a peak amplitude.

Procedure In the encoding phase, the procedure was the same as in Experiment 1. In the memory phase, the first judgment - whether the word was in the initial set (old) or not (new) - was the same. For the second judgment, participants were asked to indicate whether the person who said the word in the initial set was "Jane" or "Sid." The test phase was conducted with visually presented words, as in Experiment 1 (for a similar paradigm, see Wilding \& Rugg, 1996).

Data analysis Data were analyzed in the same way as in Experiment 1.

\section{Results}

Item recognition Accuracy and hit, miss, correct rejection, and false alarm rates across all words are shown in Table 3. Among all words, there was a significant main effect of both word type, with concrete words showing better recognition $\left(\chi^{2}(1)=\right.$ 6.77, $p=.009$ ), and confidence level, with both medium and high showing greater accuracy than low confidence $\left(\chi^{2}(2)=610.85, p<.001\right)$. The word type $\times$ confidence interaction was non-significant. Among targets, there was a main effect of confidence $\left(\chi^{2}(2)=\right.$ $961.49, p<.001)$, but not of word type. The interaction was significant $\left(\chi^{2}(2)=9.18, p=.01\right)$ at high confidence, suggesting that at greater memory strength, item recognition was worse for abstract words. Means and 95\% CIs for the main effects of word type on word and source (i.e., voice) memory are visualized in Figure 2. Finally, d' analysis revealed that after considering response sensitivity, accuracy was better for concrete concepts, $t(40)=-3.49, p=.001$.

Table 3

Mean Word Recognition Accuracy

\begin{tabular}{lcccccc}
\hline $\begin{array}{l}\text { Word } \\
\text { type }\end{array}$ & Acc & Hit & Miss & CR & FA & $\mathrm{d}^{\prime}$ \\
\hline Abs & .70 & .72 & .28 & .64 & .36 & 1.04 \\
Conc & .73 & .77 & .23 & .67 & .33 & 1.28 \\
\hline
\end{tabular}

Note. $\mathrm{CR}=$ correct rejection; FA $=$ false alarm.

Source memory Here, we again included only trials for which the word had been correctly recognized. There was a main effect of word type, with source memory for the voice context worse for abstract words $\left(\chi^{2}(1)=5.70, p=.02\right)$, as well as a main effect of confidence $\left(\chi^{2}(2)=25.22, p<.001\right)$. The interaction was non-significant. Thus, participants were again less likely to recognize the context correctly for abstract as compared to concrete words. Means and 95\% CIs are shown in Figure 2.

A
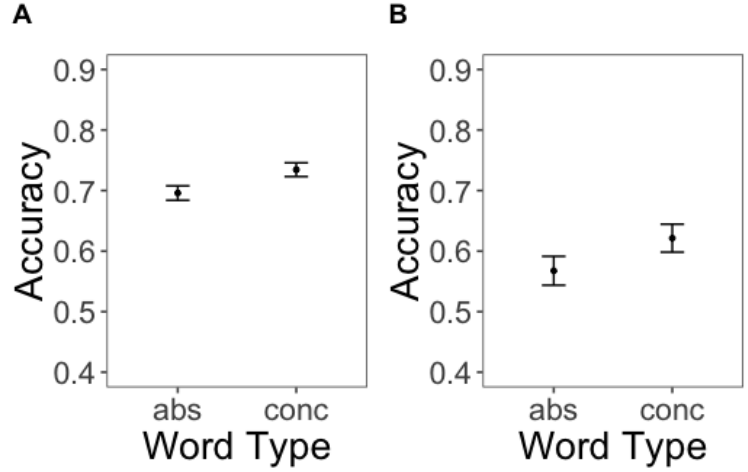

Figure 2. Effects of concreteness on (a) overall item recognition accuracy and (b) source (i.e., voice) memory.

As in Experiment 1, d' was greater for concrete than it was for abstract concepts (Table 3), and so we constructed models with d' as a predictor. A likelihood ratio test comparing the model with both $\mathrm{d}^{\prime}$ and word type versus the model with only d' was significant, $\chi^{2}(1)=5.75, p=.02$, suggesting that the effect of word type, where source memory was worse for abstract than it was for concrete concepts, was significant even after accounting for the d' concreteness advantage.

\section{Discussion}

There was again a concrete word advantage in overall item recognition. Moreover, source memory for the voice context was worse for abstract concepts. This provides support for the interpretation that the concreteness advantage also extends to episodic memory, at least for memory for simple episodic detail. However, Experiments 1 and 2 showed a baseline memory advantage for concrete words, and thus they may have been more strongly encoded, and the strength with which the words were encoded, not concreteness, may have facilitated source memory. Accordingly, we conducted a third experiment.

\section{Experiment 3}

In Experiment 3, we simplified the memory phase by instead only probing recognition memory: half of the words were presented in the same box color as they were at encoding, while half of the words were presented in a different box color. The aim here was to investigate whether there is a selective advantage in recognition memory when the context is retained in abstract concepts - that is, is recognition memory facilitated to a greater extent in abstract concepts by 
context preservation? This would suggest that while the memory trace left by abstract concepts may be weaker, it can be strengthened when context is consistent across exposures. On the other hand, if recognition memory accuracy for abstract concepts is worse when the box color at encoding is preserved at test, it would suggest that arbitrary episodic detail may be inhibited in abstract concepts.

\section{Methods}

Participants Forty UConn undergraduates with normal or corrected-to-normal vision who had not participated in Experiment 1 or 2 provided written informed consent and received course credit.

Stimuli The stimuli were the same as those in Experiments 1 and 2, and box color assignment was counterbalanced across participants.

Procedure The encoding procedure was the same as in Experiment 1. At test, participants were asked to identify as many old words as possible, ignoring the color of the box. Words were presented in the red and green boxes. Half of the words retained the box color from encoding, and half changed color.

Data analysis Item recognition data were analyzed in the same way as in Experiments 1 and 2. However, box retention (old vs. new) was used as a second fixed effect in the mixed logit model, and the interaction was word type $\times$ box retention.

\section{Results and discussion}

Accuracy and hit, miss, correct rejection, and false alarm rates across all words are shown in Table 4 . In overall old/new item recognition memory, there was a main effect of word type $\left(\chi^{2}(1)=12.29, p<.001\right)$, where memory was better for concrete words. Among targets only, there was no main effect of word type, nor was there a main effect of box retention. There was, however, an interaction between word type and box retention $\left(\chi^{2}(1)=4.92, p=.03\right.$; Figure 3$)$. Accuracy was worse when the box color was retained in abstract concepts, again operating counter to the original hypothesis, and leading to the perhaps surprising conclusion that arbitrary episodic context may even be suppressed in abstract concepts.

Table 4

Mean Item Recognition Accuracy

\begin{tabular}{lcccccc}
\hline $\begin{array}{l}\text { Word } \\
\text { type }\end{array}$ & Acc & Hit & Miss & CR & FA & d' \\
\hline $\begin{array}{l}\text { Abstract } \\
\text { Concrete }\end{array}$ & .76 & .76 & .24 & .78 & .22 & 1.59 \\
\hline
\end{tabular}

A

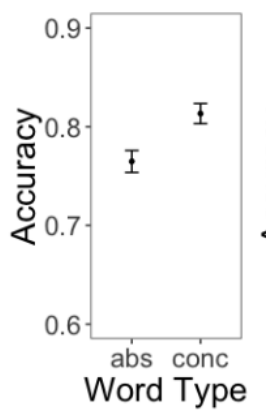

B

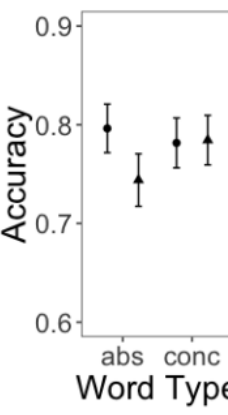

Box Retention

- new

- old

Figure 3. Plots showing (a) the main effect of concreteness on item recognition memory for all words and (b) the interaction between word type and box retention on target recognition memory accuracy (means and 95\% CIs).

\section{General Discussion}

Abstract concepts are sensitive to context, but what is the mechanism by which this sensitivity emerges? The episodic memory system was identified as a potential candidate for encoding contextual information when processing abstract concepts. In Experiments 1 and 2, however, there was a concreteness advantage for recognizing episodic contexts. In Experiment 3, context preservation conferred a disadvantage for recognizing abstract concepts, suggesting the presence of a mechanism whereby arbitrary associations are inhibited in the episodic experience(s) of the situations that activate abstract concepts.

In spite of these findings, across several literatures it is agreed that context is critical for understanding abstract concepts. However, there are differences across frameworks in terms of the type of context specified as being critical to processing abstract concepts, ranging from semantically constraining linguistic context in context-availability theory (Schwanenflugel \& Shoben, 1983), to thematic associations in the qualitatively different representations framework (Crutch \& Warrington, 2005), to meaningful situational and internal factors in grounded cognition (Barsalou \& Wiemer-Hastings, 2005). While this study sought to uncover a basic mechanism that might unify these approaches (i.e., sensitivity to episodic information), the results unequivocally ran counter to our hypothesis: there is a concreteness advantage for encoding simple episodic detail.

\section{Concreteness, context, and episodic memory}

Concreteness is a powerful organizing factor in semantic memory (e.g., De Deyne, 2017; Hollis \& Westbury, 2016), and concreteness effects are near ubiquitous in recognition memory studies. The present 
results suggest that such effects extend beyond stronger memory for concrete concepts to better associative. relational memory for concrete concepts, at least when the relation is a simple, arbitrary context. One important consideration here is the way in which we might expect context to be differentially recruited for processing concrete and abstract concepts, as this has implications for the relation between context sensitivity and concreteness.

In a review of the pervasiveness of context effects in cognition and perception, Yeh and Barsalou (2006) present two primary theses for how context affects concept processing: (1) contexts and concepts mutually activate each other, such that when processing a context, associated concepts are activated, and vice versa; and (2) when processing a concept in a particular context, properties of the concept which are relevant to that context become active. These two theses have different implications for the relation between context sensitivity and concreteness.

The first thesis resonates strongly with context availability theory, and likely suggests a concrete word advantage: concrete concepts activate contexts more strongly because they have stronger implicit ties to specific contexts. Thus, building implicit, direct associations between context and concept may have been facilitated by a similar mechanism to that which underpins context availability effects-if concrete concepts are typically associated with these sorts of contexts, then such contexts (such as boxes and voices) might be more likely to be encoded with concrete concepts.

The second thesis may be more pertinent to abstract concept processing: when processing decision in the context of your choice of beverage at $9 \mathrm{pm}$ in the local café, the activated properties will be different from when processing decision in the context of a judge determining the appropriate sentence for a felon convicted of battery. That is, the schema-based knowledge necessary in these two situations differs considerably. Decision has a number of possible interpretations, and its precise meaning - and thus, the properties activated-depends on the situation and (systematically) associated schema-based knowledge. Research on the neural dynamics underpinning schema processing (e.g., van Kesteren et al., 2013) suggests that activating these systematic associations may in fact suppress the formation of associations with arbitrary elements of an episode. This dynamic is rooted in the interplay between neural systems in medial frontal and medial temporal lobe, where medial frontal activation when processing systematic associations may dampen activation of medial temporal lobe, thereby suppressing the formation of arbitrary bindings. Exploring these neural dynamics in this paradigm is an important direction for future work.

In summary, we contend that abstract concepts activate systematic-or schema-based-contextual information, and when processing decision, the activation of schema-based information may in fact inhibit formation of arbitrary associations. This would explain why our arbitrary episodic contexts were not well remembered for abstract concepts (Experiments 1 and 2) and why context retention may have even inhibited word recognition (Experiment 3). That is, abstract concepts may be particularly sensitive to systematic or schema-based contextual constraints, implicitly activating these associations when they are absent, and thus simultaneously inhibiting arbitrary contextual associations.

\section{Limitations}

The synonym judgment task used at encoding may have worked to a disadvantage: as abstract concepts tend to have more diverse meanings, synonym judgments may be more difficult for abstract concepts, as it must be determined whether any particular sense of the word is a synonym to the target (Hoffman et al., 2013). Thus, an abstract concept like decision when paired with judgment might leave fewer resources available to process immediately available relational information (i.e., in the present study, the box color or the voice) because we must search for a context in which decision and judgment are in fact synonyms (a recent computational model makes this prediction; Popov \& Reder, 2018). Relatedly, if abstract concepts are simply more difficult to process, and the context does not help with accessing the meaning of the word, it could render the immediate context less salient. Thus, future research on context encoding in abstract and concrete concepts might benefit from departing from low-level episodic contexts. While we focused on arbitrary episodic detail, it might be fruitful to instead explore systematic contextual relations. For example, abstract concepts are thought to be represented in thematic or associative networks (e.g., faith-church), and so we might expect to see an abstract advantage in such contexts (for related evidence showing precisely this in relational vs. entity concepts, see Asmuth \& Gentner, 2017). Finally, our finding that context reinstatement did not improve item recognition even for concrete words was perplexing. This may be because we only used two contexts - context-preservation advantages may not be observed when the context is shared across too many items (Park et al., 2006). That said, with just two contexts, reinstatement still impaired item recognition for abstract words, implying that a context preservation disadvantage can be detected with only two contexts. Nevertheless, further research is 
necessary to better understand the interaction between abstract concepts and arbitrary episodic contexts.

\section{Conclusions}

This research suggests that arbitrary episodic detail is better bound with concrete than abstract concepts. Abstract concepts rely on situational context for interpretation, and given that activation of situational information is known to inhibit formation of arbitrary associations (van Kesteren et al., 2013), formation of arbitrary associations may be inhibited in abstract concepts. More broadly, the way in which the episodic memory system is recruited appears to differ as a function of concreteness, suggesting that engagement of the episodic memory system is modulated by semantic content.

\section{References}

Asmuth, J., \& Gentner, D. (2017). Relational categories are more mutable than entity categories. The Quarterly Journal of Experimental Psychology, 70(10), 2007-2025.

Balota, D. A., Yap, M. J., Hutchison, K. A., Cortese, M. J., Kessler, B., Loftis, B., ... \& Treiman, R. (2007). The English lexicon project. Behavior Research Methods, 39(3), 445-459.

Barsalou, L. W., \& Wiemer-Hastings, K. (2005). Situating abstract concepts. In D. Pecher \& R. A. Zwaan (Eds.), Grounding cognition: The role of perception and action in memory, language, and thought (pp. 129-163). Cambridge, UK: Cambridge University Press.

Brysbaert, M., Warriner, A. B., \& Kuperman, V. (2014). Concreteness ratings for 40 thousand generally known English word lemmas. Behavior Research Methods, 46(3), 904-911.

Crutch, S. J., \& Warrington, E. K. (2005). Abstract and concrete concepts have structurally different representational frameworks. Brain, 128(3), 615627.

Davachi, L. (2006). Item, context and relational episodic encoding in humans. Current Opinion in Neurobiology, 16(6), 693-700.

De Deyne, S. (2017). Mapping the lexicon using largescale empirical semantic networks. Talk presented at the Annual Meeting of the Psychonomic Society, Vancouver, BC.

Glanzer, M., \& Adams, J. K. (1985). The mirror effect in recognition memory. Memory \& Cognition, 13(1), 8-20.

Glanzer, M., \& Adams, J. K. (1990). The mirror effect in recognition memory: data and theory. Journal of Experimental Psychology: Learning, Memory, and Cognition, 16(1), 5-16.
Hoffman, P., Lambon Ralph, M. A., \& Rogers, T. T. (2013). Semantic diversity: A measure of semantic ambiguity based on variability in the contextual usage of words. Behavior Research Methods, 45(3), $718-730$.

Hollis, G., \& Westbury, C. (2016). The principals of meaning: Extracting semantic dimensions from cooccurrence models of semantics. Psychonomic Bulletin \& Review, 23(6), 1744-1756.

Kirwan, C. B., Wixted, J. T., \& Squire, L. R. (2008). Activity in the medial temporal lobe predicts memory strength, whereas activity in the prefrontal cortex predicts recollection. Journal of Neuroscience, 28(42), 10541-10548.

Paivio, A., Walsh, M., \& Bons, T. (1994). Concreteness effects on memory: When and why? Journal of Experimental Psychology: Learning, Memory, and Cognition, 20(5), 11961204.

Park, H., Arndt, J., \& Reder, L. M. (2006). A contextual interference account of distinctiveness effects in recognition. Memory \& Cognition, 34(4), 743-751.

Popov, V., \& Reder, L. (2018; preprint). Frequency effects on memory: A resource-limited theory.

Rugg, M. D., Vilberg, K. L., Mattson, J. T., Sarah, S. Y., Johnson, J. D., \& Suzuki, M. (2012). Item memory, context memory and the hippocampus: fMRI evidence. Neuropsychologia, 50(13), 30703079.

Schwanenflugel, P. J., \& Shoben, E. J. (1983). Differential context effects in the comprehension of abstract and concrete verbal materials. Journal of Experimental Psychology: Learning, Memory, and Cognition, 9(1), 82-102.

Tulving, E. (1983). Elements of episodic memory. Oxford, UK: Clarendon.

Tulving, E. (2002). Episodic memory: From mind to brain. Annual Review of Psychology, 53(1), 1-25.

Wilding, E. L., \& Rugg, M. D. (1996). An eventrelated potential study of recognition memory with and without retrieval of source. Brain, 119(3), 889905.

Yeh, W., \& Barsalou, L. W. (2006). The situated nature of concepts. The American Journal of Psychology, 119(3), 349-384.

Yonelinas, A. P. (2001). Components of episodic memory: The contribution of recollection and familiarity. Philosophical Transactions of the Royal Society of London B: Biological Sciences, 356(1413), 1363-1374.

Yonelinas, A. P. (2002). The nature of recollection and familiarity: A review of 30 years of research. Journal of Memory and Language, 46(3), 441-517. 
Yu, S. S., Johnson, J. D., \& Rugg, M. D. (2012). Hippocampal activity during recognition memory co-varies with the accuracy and confidence of source memory judgments. Hippocampus, 22(6), 1429-1437. 